\title{
Implicações biogeográficas de novos registros ornitológicos em um enclave de vegetação de campina no sudoeste da Amazônia brasileira
}

\author{
Fabíola Poletto ${ }^{1} \&$ Alexandre Aleixo ${ }^{1,2}$ \\ 1 Setor de Ornitologia, Coordenação de Zoologia, Museu Paraense Emílio Goeldi, Ministério da Ciência e Tecnologia. Caixa \\ Postal 399, 66040-170 Belém, Pará, Brasil. E-mail: fpoletto@hotmail.com \\ 2 E-mail: aleixo@museu-goeldi.br
}

\begin{abstract}
Biogeographic implications of new avian records from a patch of white-sand forest in southwestern Brazilian Amazonia. White-sand forests (WSF hereafter) include several types of stunted and open forest growing on bleached sandy soils throughout Amazonia; despite their widespread distribution, WSF occur predominantly in the upper Rio Negro drainage (NW Brazil, SE Colombia, and S Venezuela). Smaller and isolated WSF patches located in SW. Brazilian Amazonia have not been studied systematically by ornithologists; therefore, some important ornithological results obtained during a field-trip to a WSF patch in the extreme SW. corner of the state of Amazonas, centered at $7^{\circ} 22^{\prime} 33.2^{\prime \prime} \mathrm{S}$ and $73^{\circ} 00^{\prime} 42.5^{\prime \prime} \mathrm{W}$, are presented here. The first records of Hemitriccus striaticollis (Lafresnaye, 1853) (Aves, Tyrannidae) and Xenopipo atronitens Cabanis, 1847 (Aves, Pipridae) for SW. Brazilian Amazonia are presented, along with additional records of the following little known or rare species inhabiting this region: Formicivora grisea (Boddaert, 1783) (Aves, Thamnophilidae), Conopias parvus (Pelzeln, 1868) (Aves, Tyrannidae), and Heterocercus linteatus (Strickland, 1850) (Aves, Pipridae). As verified in WSF patches distributed throughout N. Peru, the WSF avifauna occurring in SW. Brazilian Amazonia is also highly influenced by species closely associated with the upper Rio Negro drainage. However, a second distinct biogeographic influence was also noted: that of species whose distribution's strongholds are located in central and E. Amazonia, S. of the Amazon river. The WSF avifauna of SW. Brazilian Amazonia is still poorly known; future ornithological surveys of isolated WSF patches in this region could lead to new range extensions and even to the discovery of unnamed taxa.

KEY WORDS. Formicivora grisea, Hemitriccus striaticollis, Conopias parvus, Xenopipo atronitens, Heterocercus linteatus.
\end{abstract}

RESUMO. Campinas constituem um tipo florestal de porte arbóreo reduzido e aspecto geral aberto, que cresce em solos arenosos e lixiviados de toda a Amazônia; apesar de sua ampla distribuição, campinas ocorrem predominantemente nas regiões do alto e médio rio Negro, no noroeste do Amazonas e sul de Roraima, incluindo também o sul da Venezuela e o sudeste da Colômbia. Existem pequenas manchas isoladas de campinas no sudoeste da Amazônia brasileira que ainda não foram estudadas sistematicamente por ornitólogos; portanto, são reportados aqui alguns resultados importantes obtidos durante uma expedição ornitológica a uma mancha de campina no extremo sudoeste do estado do Amazonas $\left(7^{\circ} 22^{\prime} 33,2^{\prime \prime S}\right.$ e $\left.73^{\circ} 00^{\prime} 42,5^{\prime \prime} \mathrm{W}\right)$. Foram documentados para região os primeiros registros das seguintes espécies: Hemitriccus striaticollis (Lafresnaye, 1853) (Aves, Tyrannidae) e Xenopipo atronitens Cabanis, 1847 (Aves, Pipridae); adicionalmente, foram obtidos registros com as seguintes espécies raras ou de distribuição pouco conhecida no sudoeste da Amazônia: Formicivora grisea (Boddaert, 1783) (Aves, Thamnophilidae), Conopias parvus (Pelzeln, 1868) (Aves, Tyrannidae) e Heterocercus linteatus (Strickland, 1850) (Aves, Pipridae). Como verificado em manchas de campina distribuídas por todo o norte do Peru, a avifauna de campina que ocorre no sudoeste da Amazônia brasileira é também altamente influenciada por espécies associadas à região do alto rio Negro. Entretanto, uma segunda influência biogeográfica também pôde ser notada: aquela de espécies cujo centro de distribuição está localizado nas campinas do centro e leste da Amazônia, ao sul do rio Amazonas. A avifauna das campinas do sudoeste da Amazônia ainda é pouco conhecida; futuros levantamentos de enclaves de campina nesta região podem levar a novas extensões de distribuiç̧ão e também à descoberta de novos táxons.

PALAVRAS CHAVE. Formicivora grisea, Hemitriccus striaticollis, Conopias parvus, Xenopipo atronitens, Heterocercus linteatus.

Campinas constituem um tipo florestal de porte arbóreo reduzido e com aspecto geral aberto, que cresce em solos arenosos e lixiviados de toda a Amazônia; já as chamadas campinaranas, crescem nos mesmos tipos de solo, mas se diferenciam das campinas principalmente pelo porte arbóreo mais elevado e pela estrutura mais densa (florestal) da vegetação (ANDERSON 1981).

Revista Brasileira de Zoologia 22 (4): 1196-1200, dezembro 2005 
Juntas, campinas e campinaranas (daqui em diante referidas em conjunto como "campinas") cobrem aproximadamente $4 \%$ do bioma Amazônico no Brasil, ocorrendo predominantemente nas regiões do alto e médio rio Negro, no noroeste do Amazonas e sul de Roraima, incluindo também o sul da Venezuela e o sudeste da Colômbia. Fora destas regiões, campinas ocorrem na forma de enclaves relativamente isolados e pequenos (CAPOBIANCo et al. 2001).

Recentemente, Alvarez \& Whitney (2003) estudaram a avifauna das campinas do departamento de Loreto, Peru, caracterizando o grau de associação de várias espécies com os ambientes de campinas. Paralelamente, levantamentos ornitológicos realizados em campinas desta região nos últimos cinco anos, resultaram na redescoberta de uma espécie, Pithys castanea Berlioz, 1948; (Aves, Thamnophilidae); ZIMMER \& IsLER 2003), na elevação de uma subespécie ao nível taxonômico de espécie, Myrmeciza castanea Zimmer, 1932; (Aves, Thamnophilidae); (IsLER et al. 2002) e na descrição de três espécies novas até então desconhecidas da ciência, Herpsilochmus gentryi Whitney \& Alvarez, 1998; (Aves, Thamnophilidae); Percnostola arenarum Isler et al. 2001; (Aves, Thamnophilidae); e Zimmerius villarejoy Alvarez \& Whitney, 2001; (Aves, Tyrannidae).

Em território brasileiro, a avifauna das campinas do sudoeste da Amazônia, definida aqui como a região compreendida a oeste do rio Madeira e ao sul do rio Solimões, não foi objeto até hoje de um estudo sistemático. Em vista desta escassez de informações sobre a avifauna das campinas do sudoeste Amazônico, são reportados aqui registros importantes obtidos para cinco espécies de aves documentadas num enclave de campina situado nesta região, sendo também discutido as implicações biogeográficas destes registros.

\section{MATERIAL E MÉTODOS}

Entre 22 e 26 de agosto de 2003, os autores participaram de um inventário rápido de avifauna num enclave de campina do sudoeste da Amazônia situado no polígono JU-008 do programa ARPA (Ministério do Meio Ambiente 2002), nos municípios limítrofes de Mâncio Lima (Acre) e Guajará (Amazonas).

Durante os trabalhos de campo foi amostrado um único transecto de quase $7 \mathrm{~km}$ de comprimento, cujo centro se situava a aproximadamente $7^{\circ} 22^{\prime} 33,2^{\prime \prime} \mathrm{S}$ e $73^{\circ} 00^{\prime} 42,5^{\prime \prime} \mathrm{W}$, no município de Guajará, Amazonas, e que incluía vários tipos fitofisionômicos de campinas. Ao longo deste transecto, foi registrada a presença de várias espécies de aves, entre elas cinco documentadas pela primeira vez ou com poucos registros para o sudoeste da Amazônia brasileira (a nomenclatura segue a recomendada pelo Comitê Brasileiro de Registros Ornitológicos 2005): Formicivora grisea (Boddaert, 1783) (Aves, Thamnophilidae), Hemitriccus striaticollis (Lafresnaye, 1853) (Aves, Tyrannidae), Conopias parvus (Pelzeln, 1868) (Aves, Tyrannidae), Xenopipo atronitens (Cabanis, 1847) (Aves, Pipridae) e Heterocercus linteatus (Strickland, 1850) (Aves, Pipridae). Essas espécies foram documentadas de duas maneiras: 1) através da gravação em fita k-7 de suas vocalizações com um gravador Sony TCM-5000 acoplado a um microfone direcional Sennheiser ME66; essas gravações serão oportunamente depositadas no Arquivo Sonoro Elias P. Coelho (ASEC) da Universidade Federal do Rio de Janeiro, Rio de Janeiro; e 2) através da coleta científica e tombamento de espécimes na coleção de aves do Museu Paraense Emílio Goeldi (MPEG), Belém, Pará.

\section{RESULTADOS E DISCUSSÃO}

A seguir, são detalhadas as circunstâncias e implicações biogeográficas dos registros obtidos com as espécies listadas abaixo.

\section{Formicivora grisea (Boddaert, 1783)}

No dia 24 de agosto de 2003, às 9:00 h da manhã, foi gravado os gritos típicos de um indivíduo desta espécie (fita "CAMPINA 1", seqüência 27, de Alexandre Aleixo) em ambiente de campina arbustiva (árvores com no máximo 5 metros de altura), situado a aproximadamente $7^{\circ} 23^{\prime} 35,9^{\prime \prime} \mathrm{S}$; $73^{\circ} 1^{\prime} 14,3$ "W no polígono JU-008; o indivíduo gravado respondeu ativamente ao play-back de suas próprias vocalizações.

Embora F. grisea seja típica de ambientes de campina e borda de mata em grande parte da Amazônia, esta espécie havia sido registrada anteriormente apenas uma única vez (em 1997) para o sudoeste da Amazônia, no estado do Amazonas, entre os quilômetros 38 e 45 da estrada Cruzeiro do Sul, Santa Bárbara ( $\left.7^{\circ} 25^{\prime} S ; 72^{\circ} 59^{\prime} \mathrm{W}\right)$, também nas campinas do polígono JU-008 e distante a apenas cerca de $30 \mathrm{~km}$ do registro apresentado aqui (ZimMer \& IsLer 2003).

Em conjunto, esse dois registros independentes e documentados através de gravações, atestam inequivocamente a existência de uma população altamente disjunta de $F$. grisea nas campinas do sudoeste Amazônico, situadas a mais de 1.000 $\mathrm{km}$ do aparente centro de distribuição conhecido da espécie, na porção leste da Amazônia.

\section{Hemitriccus striaticollis (Lafresnaye, 1853)}

No dia 24 de agosto de 2003, às 9:00 horas da manhã, foi gravado os gritos e canto de dois indivíduos desta espécie, juntamente com F. grisea (fita "CAMPINA 1", seqüência 27, de Alexandre Aleixo), que vocalizavam no estrato inferior denso de uma campina arbustiva no polígono JU-008 (7²2'35,9"S ; $73^{\circ} 1^{\prime} 14,3^{\prime \prime} \mathrm{W}$ ); um dos indivíduos gravados (presumivelmente um macho) respondeu ativamente ao play-back.

Este é o primeiro registro de $H$. striaticollis para o sudoeste da Amazônia brasileira; a localidade mais próxima para onde existem registros confirmados desta espécie é Moyobamba (a aproximadamente $500 \mathrm{~km}$ de distância), no Departamento Peruano de San Martín, onde uma outra população isolada e também associada ao ambiente de campina foi localizada (RIDGELY \& Tudor 1994). No Brasil, os registros mais próximos de $H$. striaticollis são para a bacia do rio Madeira, nos estados do Amazonas e Rondônia, a mais de $800 \mathrm{~km}$ do polígono JU-008 (Ridgely \& Tudor 1994 e espécimes depositados no MPEG). 
O aparente centro de distribuição de $H$. striaticollis compreende o Nordeste brasileiro, Brasil central e centro e leste da Amazônia, sempre ocorrendo em locais de vegetação aberta ou semi-aberta; o registro inédito reportado aqui eleva para três o número de populações isoladas desta espécie conhecidas para o oeste da Amazônia brasileira e extra-brasileira (Ridgely \& TUDOR 1994, FitzPATRick et al. 2004).

\section{Conopias parvus (Pelzeln, 1868)}

Esta é uma espécie cuja distribuição (centrada no escudo Guianense Amazônico), é bastante local ao sul do rio Amazonas e sempre associada a enclaves de campinas (Peres \& Whittaker 1991, Alvarez \& Whitney 2003). Embora C. parvus já tenha sido registrada previamente para o sudoeste Amazônico (Whittaker \& Oren 1999, Alvarez \& Whitney 2003), reportam-se aqui os primeiros espécimes desta espécie (pouco representada em coleções) obtidos para o Brasil fora do escudo Guianense.

No dia 24 de agosto de 2003, foi gravado um casal (fita "CAMPINA 1", seqüência 24, de Alexandre Aleixo) e coletado uma fêmea de C. parvus (MPEG 57486: 20g; ovário granulado 6x4 mm) no polígono JU-008 (7²3'35,9"S; 73²'14,3”W); o segundo espécime (MPEG 57740; 20.2g; testículo esquerdo 6x4 mm; crânio 100\% ossificado; terceira primária em muda), também gravado, foi obtido em 3 de novembro de 2003 num enclave de campinarana situado no sudeste do estado do Amazonas, próximo à fronteira com os estados de Rondônia e Mato Grosso, na

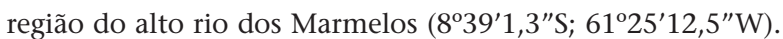

Em conjunto, esses espécimes constituem em mais uma evidência documental da distribuição ampla, porém pontual, de C. parvus por todo o sudoeste da Amazônia brasileira, sempre associada a enclaves de campinas, fato que era ignorado até recentemente (Ridgely \& Tudor 1994, Alvarez \& Whitney 2003).

\section{Xenopipo atronitens Cabanis, 1847}

Primeiro registro da espécie para o sudoeste da Amazônia; durante os trabalhos de campo no polígono JU-008 foram gravados vários indivíduos (fita "CAMPINA 1", seqüências 10a, 21 e 28, de Alexandre Aleixo) e também coletados seis espécimes de $X$. atronitens entre os dias 22 e 24 de agosto de 2003 (MPEG 57487: 16 g, ovário granulado, crânio $100 \%$ ossificado; MPEG 57488: 14g, testículo 2 x $1 \mathrm{~mm}$, crânio 100\% ossificado; MPEG 57489: $12 \mathrm{~g}$, testículo $6 x 4 \mathrm{~mm}$, crânio $100 \%$ ossificado; MPEG 57490: 12g, testículo 6x4 mm, crânio 100\% ossificado; MPEG 57491: 14g, gônadas não encontradas; crânio 100\% ossificado e MPEG 57492: 12g, testículo 6x4 mm, crânio 100\% ossificado).

A localidade mais próxima para onde $X$. atronitens havia sido registrada é Jeberos (região oeste do Departamento de Loreto, Peru), a cerca de $400 \mathrm{~km}$ do polígono JU-008; nesta localidade, uma outra população disjunta e também associada à campinas desta espécie foi descoberta em julho de 2001 (SNOw 2004). Outras populações isoladas de $X$. atronitens para o sudoeste da Amazônia incluem: Pampas Del Heath, no sudeste do Peru (Departamento de Madre de Dios; Graham et al. 1980), Serrania de Huanchaca na Bolívia (Departamento de Santa Cruz; Ridgely \&
Tudor 1994), Parque Nacional da Serra da Cutia no sudoeste do estado de Rondônia (F. Olmos, com. pess.) e município de Manicoré, Amazonas, na região do alto rio dos Marmelos ( $8^{\circ} 39^{\prime} 1,3$ "S; $\left.61^{\circ} 25^{\prime} 12,5^{\prime \prime} \mathrm{W}\right)$, onde foi coletada uma fêmea em 6 de novembro de 2003 num enclave de campinarana (MPEG 57765: 14g; ovário liso 3 × 2 mm; crânio 100\% ossificado).

As localidades de ocorrência mencionadas acima atestam que a distribuição de $X$. atronitens na porção sudoeste da Amazônia é bem mais ampla do que se supunha, sendo, contudo, pontual e restrita ao ambiente de campina (Ridgely \& Tudor 1994, SNOW 2004).

\section{Heterocercus linteatus (Strickland, 1850)}

Espécie considerada incomum e de ocorrência local e quase obrigatória em florestas de solo arenoso sazonalmente alagadas, geralmente próximas a igarapés de água preta ( et al. 1996, Alvarez \& Whitney 2003). A distribuição de H. linteatus é aparentemente mais restrita ainda no oeste Amazônico, para onde são conhecidos poucos registros, restritos à porção norte desta sub-região (Gyldenstolpe 1945, Peres \& WhitTaker 1991, Ridgely \& Tudor 1994).

Nos dias 25 e 26 de agosto de 2003 foram gravados (fita "CAMPINA 1", seqüência 38b, de Alexandre Aleixo) e coletados três espécimes de $H$. linteatus no polígono JU-008 (7²2'33,2"S; $73^{\circ} 00^{\prime} 42,5^{\prime \prime} \mathrm{W}$ ) em área de campinarana próxima a um igarapé de água preta (MPEG 57493: 24g; ovário granulado 8x3 mm, crânio 100\% ossificado; MPEG 57494: 26g; testículo 8x4 mm, crânio 100\% ossificado e MPEG 57495: 24g; testículo 8x5 mm, crânio 100\% ossificado).

O registro mais próximo do polígono JU-008 para $H$. linteatus (a aproximadamente $350 \mathrm{~km}$ ) foi feito para a localidade de Igarapé do Gordão, município de Eirunepé, Amazonas, onde apenas um único espécime foi coletado (Gyldenstolpe 1945).

Aparentemente, o registro reportado aqui é um dos mais próximos da zona de contato entre $H$. linteatus e o congênere Heterocercus aurantiivertex Sclater \& Salvin, 1880 (Aves, Pipridae), conhecido para campinas da localidade de Jenaro Herrera, Departamento de Loreto, Peru, situadas a cerca de $300 \mathrm{~km}$ a nordeste do polígono JU-008 (Alvarez \& Whitney 2003) e para o vale do Javari, em território brasileiro (СвRо 2005).

\section{Implicações biogeográficas}

O sudoeste da Amazônia (definido como a região compreendida a oeste dos rios Beni e Madeira e ao sul dos rios Marañón, Amazonas e Solimões, na Bolívia, Brasil e Peru) é a área ecologicamente mais heterogênea de toda a bacia, onde um mosaico de variedades distintas de solo determina com freqüência a ocorrência local de vários tipos de vegetação (TuOMIsTo et al. 1995). A existência de campinas no sudoeste Amazônico é determinada pela ocorrência de solos com predominância de sedimentos quartzíticos, cuja origem e idade ainda são bastante discutidos (ver revisão em Alvarez \& Whitney 2003); como nesta região solos argilosos são geralmente dominantes frente a solos arenosos, campinas aparecem sempre na forma de 
enclaves relativamente pequenos e isolados (Hookn 1994).

Do ponto de vista fitogeográfico, as campinas do polígono JU-008 (classificadas como Vegetação Lenhosa Oligotrófica dos Pântanos - arbórea aberta e arbórea densa; sensu CAPOBIANCO et al. 2001), constituem um enclave disjunto das campinas da região do alto rio Negro, situadas a mais de $700 \mathrm{~km}$, no noroeste da Amazônia (CAPobianco et al. 2001, Silveira 2003). Embora o levantamento da avifauna do polígono JU-008 tenha sido preliminar (Aleixo \& Poletto, dados não publicados), o registro de espécies típicas das campinas do alto rio Negro como Topaza pyra (Gould, 1846) (Aves, Trochilidae), Hypocnemis hypoxantha (Sclater, 1869) (Aves, Thamnophilidae) e Neopipo cinnamomea (Lawrence, 1869) (Aves, Tyrannidae), além daqueles já discutidos com Conopias parvus e Xenopipo atronitens, reforçam a conexão biogeográfica existente entre estas duas regiões, apesar de as mesmas serem separadas pelo alto rio Solimões, uma das maiores barreiras biogeográficas atuais de toda a Amazônia (HAFFER 1992).

Como mencionado anteriormente, as campinas do alto rio Negro são as mais extensas e contínuas de toda a Amazônia (CAPOBIANCO et al. 2001) e sua avifauna típica já foi caracterizada por Borges et al. (2001, 2004). Levantamentos recentes de avifauna em seis enclaves de campinas situados no Departamento de Loreto, Peru, levaram ao registro de várias populações isoladas de espécies tipicamente associadas às campinas do alto rio Negro, revelando uma conexão biogeográfica clara entre esta região e o sudoeste da Amazônia (Alvarez \& Whitney 2003).

Quando os registros discutidos aqui são colocados em perspectiva em relação aos trabalhos prévios sobre a avifauna associada à campinas na Amazônia, fica claro que a conexão biogeográfica entre as campinas do polígono JU-008 e aquelas do alto rio Negro é feita por intermédio de vários enclaves de campinas que se distribuem em forma de "arco" pelo sudeste da Colômbia e nordeste do Peru, atingindo o extremo sudoeste do território Amazônico brasileiro (Borges et al. 2001, 2004, Alvarez \& Whitney 2003). Apesar desta conexão clara, os registros reportados aqui com Formicivora grisea, Hemitriccus striaticollis e Heterocercus linteatus, além daqueles obtidos também no polígono JU-008 com Hemitriccus minimus (Todd, 1945) (Aves, Tyrannidae) (Aleixo \& Poletto, dados não publicados), revelam uma segunda influência biogeográfica nas campinas do sudoeste Amazônico: aquela de elementos típicos das campinas da Amazônia central e oriental ao sul do rio Amazonas, e que são ausentes ou têm populações marginais nas campinas do alto rio Negro. Ao que tudo indica, essa influência meridional tende a diminuir já em território Peruano, para onde não existem registros confirmados de F. grisea, por exemplo (Alvarez \& Whitney 2003, Zimmer \& IsLer 2003).

O conhecimento sobre a distribuição e composição da avifauna associada à campinas do sudoeste da Amazônia brasileira é ainda bastante incipiente; outros enclaves de campinas situados nos municípios de Tefé e Coari, também no estado do Amazonas, por exemplo, e não mapeados na base de dados de
CAPOBIANCo et al. (2001), foram amostrados preliminarmente, mas já revelaram a presença de várias espécies típicas de campinas (Peres \& WhitTaker 1991) e desconhecidas para o interflúvio Juruá e Purus. Portanto, futuros inventários de avifauna são altamente recomendáveis em campinas desta região e deverão levar ao registro de novas ocorrências de espécies raras ou distribuídas pontualmente, bem como até, a descrição de novos táxons.

\section{AGRADECIMENTOS}

O levantamento de avifauna realizado pelos autores no sudoeste do estado do Amazonas foi planejado e patrocinado, respectivamente, pela Associação SOS Amazônia e pelo Fundo Mundial para a Natureza (WWF) com o objetivo de subsidiar um documento de defesa técnica para a criação de uma unidade de conservação de proteção integral nas campinas do polígono JU-008. Verônica Passos, Maria Aparecida Oliveira Azevedo Lopes, Leôncio Menezes e guias locais foram imprescindíveis no auxílio ao trabalho de campo. Mario Cohn-Haft ajudou de modo decisivo na identificação da vocalização de Hemitriccus striaticollis. F. Poletto é bolsista de Desenvolvimento Tecnológico e Industrial (DTI) do CNPq (processo 680023/ 2004-6). A. Aleixo foi bolsista de Desenvolvimento Científico Regional (DCR) do CNPq (processo 305416/02-2) durante os trabalhos de campo e confecção do presente manuscrito e atualmente é pesquisador bolsista do convênio CNPq/SECTAM (processo 35.0415/2004-8).

\section{REFERÊNCIAS BIBLIOGRÁFICAS}

Alvarez, J. \& B.M. Whitney. 2003. New distributional records of birds from whit-sand forests of the northern Peruvian Amazon, with implications for biogeography of northern south America. The Condor, Lawrence, 105: 552-566.

Anderson, A.B. 1981. White-sand vegetation of Brazilian Amazonia. Biotropica, Fairfax, 13: 199-210.

Borges, S.H.; M. Cohn-Haft; A.M.P. Carvalães; L.M. Henriques; J.F. Pacheco \& A Whitaker. 2001. Birds of Jaú National Park, Brazilian Amazon: species check-list, biogeography and conservation. Ornitologia Neotropical, Saint Louis, 12: 109-140.

Borges, S.H. 2004. Species poor but distinct: bird assemblages in white sand vegetation in Jaú National Park, Amazonian Brazil. Ibis, London, 146: 114-124.

Capobianco, J.P.R.; A.Veríssimo; A. Moreira; D. Sawyer; I. Santos \& L.P. PINTO. 2001. Biodiversidade na Amazônia brasileira: avaliação e ações prioritárias para a conservação, uso sustentável e repartição de benefícios. São Paulo, Instituto Sócio-ambiental, 540p.

Cbro (Comitê Brasileiro de Registros Ornitológicos). 2005. Listas das aves do Brasil. Disponível em: http://www.ib.usp.br/cbro

Fitzpatrick, J.; J. Bates; K. Bostwick; I. Caballero; B. Clock; A. Farnsworth; P. Hosner; L. Joseph; G. Langham; D. Lebbin; J. Mobley; M. Robbins; E. Scholes; J. Tello; B. Walther \& K. Zimmer. 
2004. Family Tyrannidae, p. 170-463. In: J. DEL Hoyo; A. ELLIOTT $\&$ D. CHRISTIE (Eds). Handbook of the birds of the world. Barcelona, Editora Lynx, vol. 9, 863p.

Graham, G. L.; G.R. Graves; T.S. Schulenberg \& J.P. O’Neill. 1980. Seventeen bird species new to Peru from the pampas heath. The Auk, Lawrence, 97: 366-370.

Gyldenstolpe, N. 1945. The bird fauna of rio Juruá in western Brazil. Kungl. Svenska Vetenskapsakademiens Handlingar, Stockholm, 22 (3): 1-338.

Haffer, J. 1992. On the "River Effect" in some forest birds of southern Amazonia. Boletim do Museu Paraense Emílio Goeldi, Belém, 8: 217-245.

Hoorn, C. 1994. An environmental reconstruction of the palaeoAmazon river system

(Middle-Late Miocene, NW Amazonia). Paleogeography, Paleoclimatology and Paleoecology, London, 112: 187238.

Isler, M.L.; J.A. Alonso; P.R. Isler \& B.M. Whitney. 2001. A review of the Percnostola rufifrons complex (Passeriformes: Thamnophilidae) with description of a new species from northern Amazonian Peru. Wilson Bulletin, Lawrence, 113: 164-176.

Isler, M.L.; J.A. Alonso; P.R. Isler; T. Valqui; A. Begazo \& B.M. WHITNEY. 2002. Rediscovery of a cryptic species and description of a new subspecies in the Myrmeciza hemimelaena complex (Thamnophilidae) of the Neotropics. The Auk, Lawrence, 119: 362-378.

Ministério do Meio Ambiente. 2002. Programa áreas protegidas

Recebido em 11.IV.2005; aceito em 06.XII.2005. da Amazônia - ARPA. Disponível em: http://www.mma.gov. br/ppg7/doc/arpareex.pdf

Peres, C.A. \& A. WhitTaker. 1991. Annotated checklist of the bird species of the upper Rio Urucu, Amazonas, Brazil. Bulletin of the British Ornithological Club, London, 111: 156-171.

Ridgely, R. S. \& G. Tudor. 1994. The birds of South America. Austin, University of Texas Press, vol. 2, XII+814p.

Silveira, M. 2003. Vegetação e flora das campinaranas do sudoeste amazônico (JU-008). Disponível on line em: http:/ /www.nybg.org/bsci/acre/pdfs/VegetacaoeFloraCampinara nas.pdf

Snow, D. 2004. Family Pipridae, p. 110-169. In: J. DEL Hoyo; A. Elliott \& D. Christie (Eds). Handbook of the birds of the world. Barcelona, Editora Lynx, 863p.

Stotz, D.F.; J.W. Fitzpatrick; T.A. Parker III \& D.B. Moskovits. 1996. Neotropical birds: ecology and conservation. Chicago, University of Chicago Press, 478p.

Tuomisto, H.; K. Ruokolainen; R. Kalliola; A. Linna; W. Danjoy \& Z. Rodriguez. 1995. Dissecting Amazonian biodiversity. Science, Washington, 269: 63.

Whittaker, A. \& D.C. Oren. 1999. Important ornithological records from de rio Juruá, western Amazonia, including twelve additions to the Brazilian avifauna. Bulletin of the British Ornithological Club, London, 119 (4): 235-260.

Zimmer, K. \& M.L. IsLer. 2003. Family Thamnophilidae, p. 358447. In: J. Del Hoyo; A. Elliott \& D. Christie (Eds). Handbook of the birds of the world. Barcelona, Editora Lynx, vol. 8, $845 \mathrm{p}$. 